\title{
An optical biomarker of hypoxic- ischaemic injury severity in the neonatal brain
}

Tachtsidis, Ilias, Lange, Frédéric, Pinti, Paola, Bale, Gemma, Sokolska, Magdalena, et al.

Ilias Tachtsidis, Frédéric Lange, Paola Pinti, Gemma Bale, Magdalena Sokolska, Adnan Avdic-Belltheus, Christopher Meehan, Nicola Robertson, Shahrzad Parsa, Udo Weigel, Turgut Durduran, Subhrabrata Mitra, "An optical biomarker of hypoxic-ischaemic injury severity in the neonatal brain," Proc. SPIE 11920, Diffuse Optical Spectroscopy and Imaging VIII, 119200I (9 December 2021); doi: 10.1117/12.2615226

SPIE. Event: European Conferences on Biomedical Optics, 2021, Online Only 


\title{
An optical biomarker of hypoxic-ischaemic injury severity in the neonatal brain
}

\author{
Ilias Tachtsidis ${ }^{\mathrm{a}}$, Frédéric Langea, Paola Pintia, g, Gemma Bale, ${ }^{\mathrm{a}, \mathrm{f}}$, Magdalena Sokolska ${ }^{\mathrm{b}}$, Adnan Avdic- \\ Belltheus $^{c}$, Christopher Meehan ${ }^{\mathrm{c}}$, Nicola Robertson ${ }^{\mathrm{c}}$, Shahrzad Parsa ${ }^{\mathrm{d}}$, Udo Weigel ${ }^{\mathrm{d}}$, Turgut Durduran ${ }^{\mathrm{e}}$, and \\ Subhrabrata Mitra ${ }^{\mathrm{c}}$ \\ ${ }^{a}$ Department of Medical Physics and Biomedical Engineering, University College London, Gower Street, London WC1E 6BT, UK, \\ i.tachtsidis@ucl.ac.uk ${ }^{b}$ Medical Physics and Biomedical Engineering, University College London NHS Foundation Trust, London, UK, ${ }^{c}$ Institute

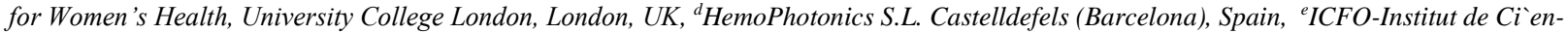 \\ cies Fot'oniques, The Barcelona Institute of Science and Technology, Castelldefels \\ (Barcelona), Spain, ${ }^{f}$ Department of Physics and Department of Engineering, University of Cambridge, Cambridge, UK, ${ }^{g}$ Centre for Brain and \\ Cognitive Development, ToddlerLab CAVE, Birkbeck, London, UK
}

Abstract: We present a new optical platform that combines broadband near-infrared spectroscopy and diffuse correlation spectroscopy for identification of brain injury severity in a preclinical model of hypoxic-ischemic encephalopathy of the neonatal brain.

\section{Introduction}

Perinatal injury to the developing brain continues to remain a significant cause of neonatal morbidity and mortality. Each year, 0.421-1.054 million children die globally following intrapartum complications. Therapeutic hypothermia improves neurodevelopmental outcome following hypoxic ischaemic encephalopathy (HIE), but still 40-79\% of cooled infants die or develop significant disability following HIE[1]. The pathophysiology of newborn brain injury following hypoxia-ischaemia (HI) is complex and our current understanding leading to therapeutic interventions are based on the findings of cerebral metabolic changes and neural recovery following HIE, based on bioenergetics changes noted on MR spectroscopy. The availability of early cot-side markers of neuronal injury that correlate with disease severity and are predictive of neurodevelopmental disability in childhood would likely to facilitate a more targeted therapeutic approach using adjunctive therapies. There is an urgent clinical need to identify the infants at most risk as early as possible after birth, who may benefit from adjunct therapies and/or redirection of clinical care for effective rehabilitation. Early detection and assessment of brain neurological status requires sensitive, robust and easy to measure cot side biomarkers[2]. To answer this need we developed a photonics platform that fuses broadband near-infrared spectroscopy (bNIRS) and diffuse correlation spectroscopy (DCS), allowing the synchronous measurement of (i) haemoglobin oxygenation changes, (ii) mitochondrial function through measurements of the oxidation state of cytochrome-c-oxidase (oxCCO) and (iii) cerebral blood flow index (BFi). We have previously demonstrated the strength of combining these measurements in understanding the physiological responses during $\mathrm{HI}$ in the neonatal preclinical model[3]. The aim of this current study is to demonstrate in a newborn preclinical model of HI injury how the combination of these measurements can offer a biomarker of brain injury severity.

\section{Methods and materials}

\subsection{The bNIRS-DCS system (Florence)}

The Florence system is composed of both a bNIRS and a DCS instruments. The bNIRS consists of a tungsten halogen lamp light source (HL-2000-FHSA, Ocean Optics, USA) with a 700nm longpass filter, and a custom-made micro spectrometer (644917nm, cooled CCD, 1024 pixels, Wasatch Photonics, USA). The DCS system (custom-made by Hemophotonics), consists of a $785 \mathrm{~nm}$ long coherence $(>8 \mathrm{~m})$ diode laser and a 4 channel APD detector. To guide the light to the piglet, a multimode fibre (400 $\mu \mathrm{m}, 0.37 \mathrm{NA}$ ) is used for the DCS laser, and a fibre bundle of 2.5 $\mathrm{mm}(30 \mu \mathrm{m}$ fibres, $0.55 \mathrm{NA})$ is used for the bNIRS halogen source. For the detection, a single fibre bundle is used to direct the light to the 4 APDs (4 single mode fibres, $3.5 \mu \mathrm{m}, 0.13 \mathrm{NA}$ ) for the DCS, and to bNIRS ( $2 \mathrm{~mm}$ bundle of $30 \mu \mathrm{m}$ fibres, $0.55 \mathrm{NA}$ ). All the fibres were made by Fiberoptic Sytems, USA and were 3 meters long. A custom 3D printed probe holder was designed to securely

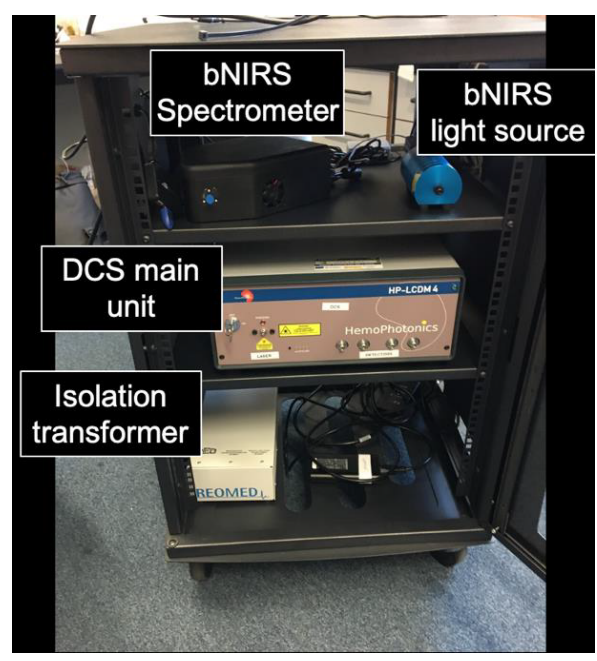

Fig. 1. Picture of the Florence device. 
attach the fibres on the head of the piglet; with a source-detector separation of $3 \mathrm{~cm}$ and $2 \mathrm{~cm}$ for the bNIRS and the DCS respectively. We use computer-controlled shutters to time multiplex the two instruments, allowing fast sequential measurements from each modality. All the components of the system are mounted on a trolley to facilitate its use in a clinical environment, and a picture of the device can be found in figure 1. The Florence measurements include the changes in concentration of oxy- and deoxy- haemoglobin $\left(\mathrm{HbO}_{2}, \mathrm{HHb}\right)$ from which we can derive brain oxygenation changes $\left(\mathrm{HbDiff}=\mathrm{HbO}_{2}-\mathrm{HHb}\right)$ and brain blood volume changes $\left(\mathrm{HbT}=\mathrm{HbO}_{2}+\mathrm{HHb}\right)$; the changes in the oxidation state of cytochrome-c-oxidase (oxCCO); and the cerebral blood flow index (BFi).

\subsection{Study protocol}

Male Large White piglets aged $\leq 36 \mathrm{~h}$ were sedated with intramuscular midazolam $(150 \mu \mathrm{g})$ and anaesthetized with 2$3 \% \mathrm{v} / \mathrm{v}$ isoflurane. Following this umbilical arterial and venous catheters were inserted for continuous monitoring of systemic physiology and inflatable occluders were placed around both common carotid arteries. This study was conducted in accordance with the UK Home Office Guidelines [Animals (Scientific procedures) Act, 1986] and complies with ARRIVE guidelines. The Ethics Committee of University College London approved the study. The probes of the Florence system were then securely placed on top of the piglet head. Following a short baseline period (10 min), we induced a transient HI by remote occlusion of both common-carotid arteries, and with simultaneous reduction of fractional inspired oxygen $\left(\mathrm{FiO}_{2}\right)$ in steps down to 6-9\%. The $\mathrm{HI}$ period was adjusted to either 20min for a mild injury $(n=7)$ or $25 \mathrm{~min}$ for a severe injury $(n=7)$. Following the HI period, occluders were deflated and $\mathrm{FiO}_{2}$ was normalised (recovery period).

\subsection{Analysis}

The nadir/peak point and area under the curve (AUC) during the insult period were extracted from the Florence measurements after a Z-transform (mean-variance-normalisation). We then estimated the relative power (rPWR) and the relative cost (rCST) by performing a $45^{\circ}$ rotation of the (1) oxygenation (HbDiff)-metabolism(oxCCO); (2) blood volume(HbT)-metabolism(oxCCO); (3) blood flow(BFi)-metabolism(oxCCO); (4) oxygenation(HbDiff)-blood flow(BFi) and (5) blood volume(HbT)-blood flow(BFi). The rPWR and rCST analysis method is described in reference [4]. An example of the transformation equation for HbDiff-oxCCO is shown below Eq 1. In our case, when analysing $\mathrm{BFi}$-oxCCO this procedure generates (i) a rPWR axis, where the positive and negative ends correspond to high perfusion+high metabolism and low perfusion+low metabolism respectively; (ii) a rCST axis perpendicular to the rPWR, where the positive and negative ends correspond to high metabolism+low perfusion and low metabolism+high perfusion repetitively. Due to the nature of the HI insult we expect our measurements to cluster at the negative ends of the rPWR axis.

$$
\left[\begin{array}{c}
r P W R \\
r C S T
\end{array}\right]=\left[\begin{array}{cc}
\cos \left(45^{\circ}\right) & \sin \left(45^{\circ}\right) \\
-\sin \left(45^{\circ}\right) & \cos \left(45^{\circ}\right)
\end{array}\right]\left[\begin{array}{c}
z(\text { HbDiff }) \\
z(\text { oxCCO })
\end{array}\right]
$$

A two-sample student's t-test was then performed on rPWRs and rCSTs to test for significant differences $(\mathrm{p}<0.05)$ between the mild and the severe group.

\section{Results}

A summary of the rPWR group results is shown in Fig. 2. We found significant differences in the rPWR both for the nadir/peak point and the AUC between the mild and severe groups for oxygenation versus metabolism (HbDiff- oxCCO),
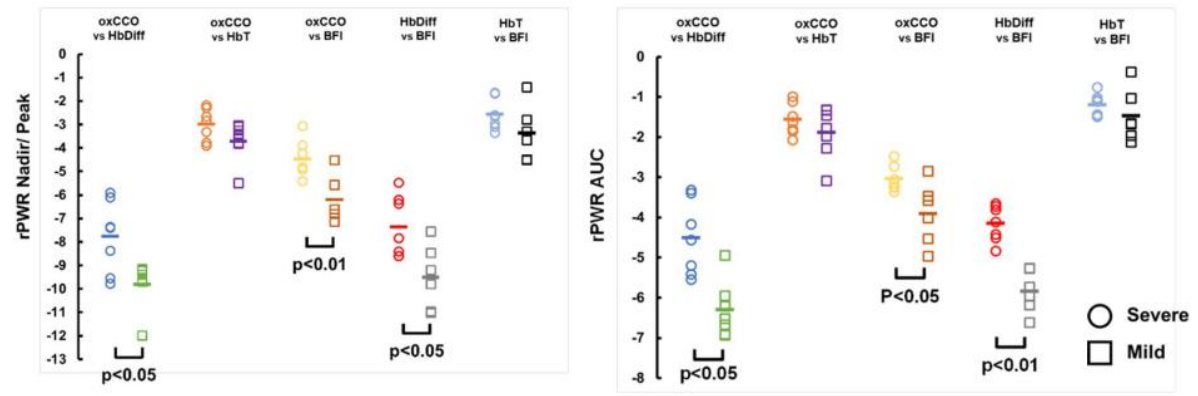

Fig. 2. Severe versus mild group results for relative power both nadir/peak and AUC. Each point is a piglet.

blood flow versus metabolism (BFi-oxCCO) and oxygenation versus blood flow (HbDiff-BFi). In all instances, the rPWR was lower, more negative in the mild versus the severe group. The rCST results demonstrated significance for 

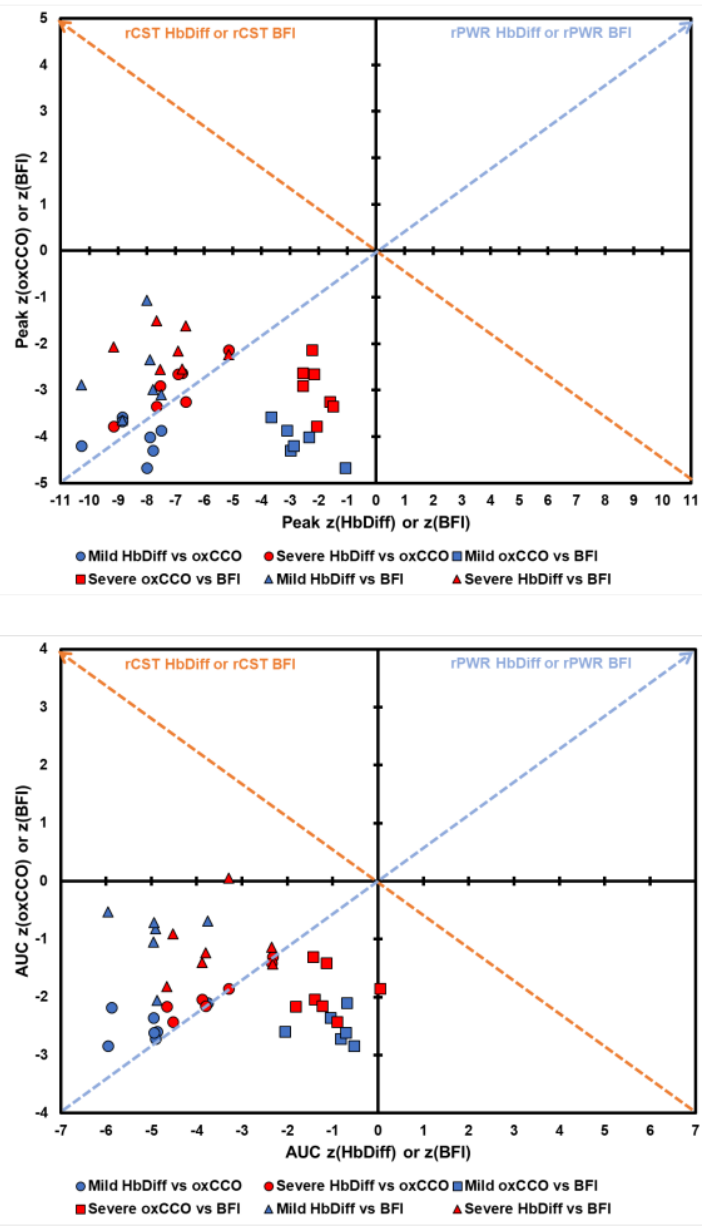

Fig. 3. Visual representation of rPWR and rCST for the Peak/Nadir (top) and AUC (bottom) zvalues. Each point is a piglet. the (i) nadir/peak point only for HbT-oxCCO and HbT-BFi and (ii) AUC only for HbDiff-oxCCO and HbT-oxCCO. Visual representation of the z-values for the peak/nadir and AUC for HbDiff, oxCCO and BFi are shown in Fig. 3. As demonstrated during the insult period we expect a drop/reduction in oxygenation, cerebral blood flow and metabolism; therefore z-scores are clustered at the bottom left corner of the diagrams in Fig. 3. In addition, it is visually clear that piglets in the mild group demonstrated larger drops in the $\mathrm{z}$-score that is why the severe group piglets are closer to the center of the diagrams

\section{Conclusion}

We present the Florence instrument, which combines bNIRS and DCS, and demonstrated that the rPWR estimation that combines measurements from both bNIRS and DCS has the capacity to distinguish the mild from the severe HI piglet group. The relative power reflects the extent of concurrent supply of oxygenation(HbDiff)/perfusion(BFi) and utilization (oxCCO). During the insult, oxygenation, metabolism and perfusion drop; however, the mild group experiences a larger proportional drop in these measurements when compared to the severe group. We hypothesize that in the mild group the changes in oxygenation or energy supply versus utilization occurs in a larger extent and with a higher degree of proportionality.

\section{References}

[1].Robertson, C.M. and M. Perlman, Follow-up of the term infant after hypoxic-ischemic encephalopathy. Paediatr Child Health, 2006. 11(5): p. 278-82.

[2.]Ferriero, D.M. and S.L. Bonifacio, The search continues for the elusive biomarkers of neonatal brain injury. J Pediatr, 2014. 164(3): p. 438-40. [3.]Rajaram, A., et al., Simultaneous monitoring of cerebral perfusion and cytochrome $c$ oxidase by combining broadband near-infrared spectroscopy and diffuse correlation spectroscopy. Biomed Opt Express, 2018. 9(6): p. 2588-2603.

[4.]Shokri-Kojori, E., et al., Correspondence between cerebral glucose metabolism and BOLD reveals relative power and cost in human brain. Nat Commun, 2019. 10(1): p. 690. 